\title{
A FITTED MESH METHOD FOR A CLASS OF SINGULARLY PERTURBED PARABOLIC PROBLEMS WITH A BOUNDARY TURNING POINT $^{1}$
}

\author{
RAYMOND K. DUNNE AND EUGENE O'RIORDAN \\ School of Mathematical Sciences, Dublin City University \\ Dublin 9, Ireland
}

\section{GRIGORII I. SHISHKIN}

Institute of Mathematics and Mechanics, Ural Branch of the Russian Academy of Sciences 16 S. Kovalevskaya Str., 620219 Ekaterinburg, Russia

Dedicated to John J.H.Miller on the occasion of his 65th birthday.

\begin{abstract}
A class of singularly perturbed time-dependent convection-diffusion problems with a boundary turning point is examined on a rectangular domain. The solution of problems from this class possesses a parabolic boundary layer in the neighborhood of one of the sides of the domain. Classical numerical methods on uniform meshes are known to be inadequate for problems with boundary layers. A numerical method consisting of a standard upwind finite difference operator on a fitted mesh is constructed. It is proved that the numerical approximations generated by this method converge uniformly with respect to the singular perturbation parameter. Numerical results are presented that verify computationally the theoretical result.
\end{abstract}

2000 Mathematics Subject Classification: 65M06, 65M12, 65M15.

Keywords: singular perturbation, piecewise-uniform mesh, boundary turning point, finite-difference scheme.

\section{Introduction}

Consider the following class of singularly perturbed parabolic problems:

$$
\left(P_{\varepsilon, p}\right)\left\{\begin{aligned}
L_{\varepsilon} u(x, t) \equiv\left(\varepsilon u_{x x}+a u_{x}-b u_{t}-d u\right)(x, t)=f(x, t) & \text { in } D, \\
u(x, t)=g(x, t) & \text { on } \Gamma, \\
a(x, t)=a_{0}(x, t) x^{p}, \quad p \geqslant 1 \quad \forall(x, t) \in \bar{D}, \quad a_{0}(x, t) \geqslant & \alpha>0, \\
b(x, t) \geqslant \beta>0, \quad d(x, t) \geqslant \delta \geqslant 0 \quad \forall(x, t) \in & \bar{D},
\end{aligned}\right.
$$

\footnotetext{
${ }^{1}$ This research was supported in part by the National Center for Plasma Science and Technology Ireland, by the Albert College Fellowship scheme of Dublin City University, by the Enterprise Ireland grant SC2000-070 and by the Russian Foundation for Basic Research under grant No. 01-01-01022.
} 
where

$$
\begin{aligned}
D & =\Omega \times(0, T], \quad \Omega=(0,1), \\
\Gamma & =\bar{D} \backslash D=\Gamma_{l} \cup \Gamma_{b} \cup \Gamma_{r}, \\
\Gamma_{l} & =\{(0, t) \mid 0 \leqslant t \leqslant T\}, \\
\Gamma_{b} & =\{(x, 0) \mid 0 \leqslant x \leqslant 1\}, \\
\Gamma_{r} & =\{(1, t) \mid 0 \leqslant t \leqslant T\} .
\end{aligned}
$$

We assume that $a_{0}, b, d, f$, and $g$ are sufficiently regular and that $f$ and $g$ satisfy sufficient compatibility conditions at the corners of the domain.

In general the solutions of problems from the above class possess a boundary layer (nature and location specified below), when the singular perturbation parameter $\varepsilon$ is small (see, for example, $[7,9])$. It is well known that numerical methods using standard finite difference operators on uniform meshes are inadequate for solving problems with boundary layers (see [2]). In this paper we construct a method consisting of a standard upwind finite difference operator on a piecewise-uniform fitted mesh. We require that our method be pointwiseaccurate and $\varepsilon$-uniform. That is, we require that

$$
\left\|U^{N}-u\right\|_{\overline{D^{N}}} \leqslant C N^{-q}, \quad q>0,
$$

where $U^{N}$ is the numerical solution computed on the mesh $D^{N}$ and $u$ is the exact solution. Note that in this paper $C, C_{1}, C_{2}$, and $C_{3}$ are positive constants independent of $\varepsilon$ and the discretization parameter $N$. The norm, $\|\cdot\|$ (sometimes subscripted), used throughout is the pointwise maximum norm.

Problem (1.1) is the so-called boundary turning point problem. For $p>1$, the turning point is called a multiple turning point. It is a parabolic partial differential equation where the coefficient of the convective term is zero along certain parts of the boundary of the domain. Note that we make no assertions about a steady-state solution of this problem. Hence, the work of [13] is not relevant to this paper. The corresponding reduced problem is defined to be

$$
\begin{aligned}
\left(a\left(v_{0}\right)_{x}-b\left(v_{0}\right)_{t}-d v_{0}\right)(x, t)=f(x, t) & \text { in } D, \\
v_{0}(x, t)=g(x, t) & \text { on } \Gamma_{b} \cup \Gamma_{r} .
\end{aligned}
$$

The solution is said to have a parabolic boundary layer in the neighborhood of $\Gamma_{l}$ when $a(0, t)=0$ and $b(0, t)>0$, as the boundary $x=0$ is then a characteristic curve of the reduced problem. If $a(x, t) \geqslant \alpha>0$, then every characteristic curve of the reduced hyperbolic problem (1.2) intersects the boundary $\Gamma_{l}$ at one point. The character of the resulting boundary layer function is one-dimensional and results from the discrepancy between the value being carried along the characteristic curve and the value of the boundary data $g(0, t)$ at the point of intersection. Such a boundary layer function is called a regular (or exponential) boundary layer function (see [2]). On the other hand, if $a(x, t) \equiv 0$ at all points of $\bar{D}$, then the characteristic curves are all parallel to the boundary $\Gamma_{l}$. In this case, the boundary data $g(0, t)$ at all points of $\Gamma_{l}$ determine the character of the resulting boundary layer function. Such boundary layer functions are called parabolic boundary layer functions. In this paper, the characteristics of the reduced problem (1.2) do not intersect the boundary $\Gamma_{l}$, but they deviate increasingly from the vertical away from the lateral boundary. This is a novel feature relating to the turning point nature of singularly perturbed parabolic problems for the problem class being examined in this paper. 
The analysis in this paper uses a suitable decomposition of the error into a regular and singular component and a comparison principle combined with appropriate barrier functions. Using this decomposition and estimates on the singular component yields error bounds in space of the form $C N^{-1}(\ln N)^{2}$. By assuming more regularity of the data, error bounds in space of the form $C N^{-1} \ln N$ could be obtained using sharper estimates on the derivatives of the singular component. This analysis (when $p$ is set equal to 0 ) is also valid for the case

$$
a(x, t) \geqslant \alpha>0 \quad \forall(x, t) \in \bar{D} .
$$

The solutions of problems from the class then possess a different type of layer than the layers examined in this paper, viz., a regular layer. This class of problems has been extensively studied (see, for example, $[3,10,11]$ ). Moreover, the method of proof given in this paper can also be applied to the reaction-diffusion case, when $a$ is identically zero (see [8]). In this case the solutions of problems from the class possess parabolic boundary layers at both $x=0$ and $x=1$. These results indicate that the technique of proof used in this paper can be applied to a wide variety of problems involving boundary layers. This illustrates the potential of the technique in establishing theoretical results for other classes of singularly perturbed problems.

It is worth mentioning that problems from the class $P_{\varepsilon, p}$ can arise from considering convection-diffusion problems posed on nonrectangular domains (see Dunne et al. [1] for some examples). This fact motivated the present authors' study of the problem. Ordinary differential equations of a form related to (1.1) have been dealt with by several authors (see, for example, $[5,6,12,13])$ and arise in geophysics and in modeling thermal boundary layers in laminar flow (see [12] and the references therein).

In Section 2 we consider some properties of the continuous problem. In particular, we establish a maximum principle and bounds on the derivatives of the solution. In Section 3 we obtain sharper bounds on the derivatives through a suitable decomposition of the solution into regular and layer components. The numerical method is constructed in Section 4 and its monotonicity is proved. In Section 5 we show that the numerical solutions generated by the method converge uniformly to the solution of the continuous problem with respect to the singular perturbation parameter. Finally, numerical results are presented in Section 6 .

\section{The continuous problem}

The differential operator $L_{\varepsilon}$ in (1.1) satisfies the following minimum principle.

Lemma 2.1 (Minimum Principle). Let $v \in C^{2,1}(\bar{D})$. If $v(x, t) \geqslant 0 \forall(x, t) \in \Gamma$ and $L_{\varepsilon} v(x, t) \leqslant 0 \forall(x, t) \in D$, then $v(x, t) \geqslant 0 \forall(x, t) \in \bar{D}$.

An immediate consequence of this is the following bound on the solution of any problem from $P_{\varepsilon, p}$.

Lemma 2.2. Let $u$ be the solution of (1.1). Then the following bound holds:

$$
\|u\|_{\bar{D}} \leqslant\|g\|_{\Gamma}+\frac{T}{\beta}\|f\|_{\bar{D}} .
$$

We will now assume the following bounds on the derivatives of the solution of any problem from $P_{\varepsilon, p}$. 
Assumption 1. For all nonnegative integers $i, j$, such that $0 \leqslant i+2 j \leqslant 3$,

$$
\left\|\frac{\partial^{i+j} u}{\partial x^{i} \partial t^{j}}\right\|_{D} \leqslant C \varepsilon^{-i / 2} .
$$

These bounds are motivated by the following heuristic argument: we transform the independent variable $x$ to the stretched variable $\hat{x}=x / \sqrt{\varepsilon}$. The transformed problem class $\hat{P}_{\varepsilon, p}$ is then

$$
\left(\hat{P}_{\varepsilon, p}\right) \quad\left\{\begin{aligned}
\left(\hat{u}_{\hat{x} \hat{x}}+\hat{a} \varepsilon^{\frac{p-1}{2}} \hat{u}_{\hat{x}}-\hat{b} \hat{u}_{t}-\hat{d} \hat{u}\right)(\hat{x}, t)=\hat{f}(\hat{x}, t) & \text { in } \hat{D} \\
\hat{u}(\hat{x}, t)=\hat{g}(\hat{x}, t) & \text { on } \hat{\Gamma},
\end{aligned}\right.
$$

where $\hat{D}=(0,1 / \sqrt{\varepsilon}) \times(0, T]$ and $\hat{\Gamma}$ is its boundary. For $p>1$ we neglect the term involving $\varepsilon^{\frac{p-1}{2}}$ which is small and we get a differential equation independent of $\varepsilon$ and therefore we must have

$$
\left\|\frac{\partial^{i+j} \hat{u}}{\partial \hat{x}^{i} \partial t^{j}}\right\|_{\hat{D}} \leqslant C
$$

Returning back to the original variable, we get (2.1). If $p=1$, we retain all terms in the differential equation. As the equation is then independent of $\varepsilon$, the same argument applies.

Remark 2.1. The bound (2.1) could be established using the techniques of [10] based on [4] (see also [8]).

We shall also assume the following which essentially states that for any problem from $P_{\varepsilon, p}$ we can always choose the boundary condition on $\Gamma_{l}$ so that we get no boundary layer in the solution of the problem.

Assumption 2. For any problem from the class $P_{\varepsilon, p}$ there exists a function $h(t)$ such that the solution to the problem

$$
\begin{aligned}
L_{\varepsilon} v(x, t) & =f(x, t) & & \text { in } D, \\
v(x, t) & =g(x, t) & & \text { on } \Gamma_{b} \cup \Gamma_{r}, \\
v(x, t) & =h(t) & & \text { on } \Gamma_{l}
\end{aligned}
$$

satisfies the following: for all nonnegative integers $i, j$, such that $0 \leqslant i+2 j \leqslant 3$,

$$
\left\|\frac{\partial^{i+j} v}{\partial x^{i} \partial t^{j}}\right\|_{D} \leqslant C\left(1+\varepsilon^{2-i / 2}\right) .
$$

\section{Decomposition of solution}

Consider the following decomposition of the solution into a regular and a singular part. Let

$$
u(x, t)=v(x, t)+w(x, t) \quad \forall(x, t) \in \bar{D},
$$

where

$$
\begin{aligned}
L_{\varepsilon} v(x, t) & =f(x, t) & & \text { in } D, \\
v(x, t) & =u(x, t) & & \text { on } \Gamma_{b} \cup \Gamma_{r}, \\
v(x, t) & =h(t) & & \text { on } \Gamma_{l}
\end{aligned}
$$


with $h$ chosen so that $(2.2)$ is satisfied. Thus, $w$ is the solution to the problem

$$
\begin{aligned}
L_{\varepsilon} w(x, t) & =0 & & \text { in } D, \\
w(x, t) & =0 & & \text { on } \Gamma_{b} \cup \Gamma_{r}, \\
w(x, t) & =u(x, t)-v(x, t) & & \text { on } \Gamma_{l} .
\end{aligned}
$$

We now derive a sharp bound on $w$, the part of the decomposition that represents the boundary layer. This bound is given in the following theorem.

Theorem 3.1. Let $w$ be the solution of (3.2). Then

$$
|w(x, t)| \leqslant C e^{-x / \sqrt{\varepsilon}} \quad \forall(x, t) \in \bar{D}
$$

Proof. Consider the barrier functions

$$
\psi_{1}^{ \pm}(x, t)=C e^{-x / \sqrt{\varepsilon}} e^{A t} \pm w(x, t),
$$

where $A=\max _{\bar{D}}\{0,(1-d) / b\}$. These functions satisfy the hypotheses of Lemma 2.1 and therefore

$$
\psi_{1}^{ \pm}(x, t) \geqslant 0 \quad \forall(x, t) \in \bar{D}
$$

\section{Formulation of the numerical method}

We now introduce the appropriate discretization that we will use for generating numerical approximations to problems from the class $P_{\varepsilon, p}$. This consists of a standard upwind finite difference operator on a fitted piecewise uniform mesh. The difference operator $L_{\varepsilon}^{N}$ on a mesh $D^{N}$ is defined for any mesh function $Z^{N}$ as

$$
L_{\varepsilon}^{N} Z^{N}\left(x_{i}, t_{j}\right) \equiv\left(\varepsilon \delta_{x}^{2} Z^{N}+a D_{x}^{+} Z^{N}-b D_{t}^{-} Z^{N}-d Z^{N}\right)\left(x_{i}, t_{j}\right) \quad \forall\left(x_{i}, t_{j}\right) \in D^{N} .
$$

Define the mesh $\overline{\Omega_{u}^{N_{t}}}$ that discretizes $[0, T]$ with $N_{t}$ uniform mesh elements as

$$
\overline{\Omega_{u}^{N_{t}}}=\left\{t_{j} \mid t_{j}=T j / N_{t}, \quad 0 \leqslant j \leqslant N_{t}\right\}
$$

and the piecewise-uniform mesh $\overline{\Omega_{\sigma}^{N_{x}}}$ that discretizes $\bar{\Omega}=[0,1]$ with $N_{x}$ mesh elements as

$$
\overline{\Omega_{\sigma}^{N_{x}}}=\left\{x_{i} \mid x_{i}=\left\{\begin{array}{lc}
2 i \sigma / N_{x}, & 0 \leqslant i \leqslant N_{x} / 2, \\
\sigma+2\left(i-N_{x} / 2\right)(1-\sigma) / N_{x}, & N_{x} / 2<i \leqslant N_{x}
\end{array}\right\}\right.
$$

where

$$
\sigma=\min \left\{\frac{1}{2}, \sqrt{\varepsilon} \ln N_{x}\right\} .
$$

It can be seen that $\Omega_{\sigma}^{N_{x}}$ consists of two uniform meshes, with $N_{x} / 2$ mesh elements in each, joined together at the transition point $\sigma$. When $\sigma=1 / 2$, the mesh is uniform, otherwise the mesh condenses near $\Gamma_{l}$. We use the notation $N=\left(N_{x}, N_{t}\right)$ and define the resulting piecewise uniform fitted mesh to be the tensor product

$$
D_{\sigma}^{N}=\Omega_{\sigma}^{N_{x}} \times \Omega_{u}^{N_{t}}
$$


and its boundary points $\Gamma_{\sigma}^{N}$ are $\Gamma_{\sigma}^{N}=\overline{D_{\sigma}^{N}} \cap \Gamma$. The resulting fitted mesh finite difference method is then

$$
\begin{aligned}
L_{\varepsilon}^{N} U^{N}\left(x_{i}, t_{j}\right) & =f\left(x_{i}, t_{j}\right) \text { in } D_{\sigma}^{N}, \\
U^{N}\left(x_{i}, t_{j}\right) & =g\left(x_{i}, t_{j}\right) \text { on } \Gamma_{\sigma}^{N} .
\end{aligned}
$$

We now state a discrete analogue of the minimum principle given in Lemma 2.1.

Lemma 4.1 (Discrete Minimum Principle). Let $Z^{N}$ be any mesh function defined on $\overline{D_{\sigma}^{N}}$. If $Z^{N}\left(x_{i}, t_{j}\right) \geqslant 0 \quad \forall\left(x_{i}, t_{j}\right) \in \Gamma_{\sigma}^{N}$ and $L_{\varepsilon}^{N} Z^{N}\left(x_{i}, t_{j}\right) \leqslant 0 \quad \forall\left(x_{i}, t_{j}\right) \in D_{\sigma}^{N}$, then $Z^{N}\left(x_{i}, t_{j}\right) \geqslant 0 \quad \forall\left(x_{i}, t_{j}\right) \in \overline{D_{\sigma}^{N}}$. $L_{\varepsilon}^{N}$.

A consequence of this is the following stability property of the finite difference operator

Lemma 4.2. Let $Z^{N}$ be any mesh function defined on $\overline{D_{\sigma}^{N}}$. If

$$
Z^{N}\left(x_{i}, t_{j}\right) \geqslant 0 \quad \forall\left(x_{i}, t_{j}\right) \in \Gamma_{\sigma}^{N},
$$

then

$$
\left|Z^{N}\left(x_{i}, t_{j}\right)\right| \leqslant \max _{\Gamma_{\sigma}^{N}}\left|Z^{N}\right|+T \max _{D_{\sigma}^{N}}\left|L_{\varepsilon}^{N} Z^{N}\right| / \beta \quad \forall\left(x_{i}, t_{j}\right) \in \overline{D_{\sigma}^{N}} .
$$

\section{Decomposition of numerical solution and error estimates}

In the way analogous to the continuous case we decompose our numerical solution into a regular and a singular part. Let

$$
U^{N}\left(x_{i}, t_{j}\right)=V^{N}\left(x_{i}, t_{j}\right)+W^{N}\left(x_{i}, t_{j}\right) \quad \forall\left(x_{i}, t_{j}\right) \in \overline{D_{\sigma}^{N}},
$$

where

$$
\begin{aligned}
& L_{\varepsilon}^{N} V^{N}\left(x_{i}, t_{j}\right)=f\left(x_{i}, t_{j}\right) \text { in } D_{\sigma}^{N}, \\
& V^{N}\left(x_{i}, t_{j}\right)=v\left(x_{i}, t_{j}\right) \text { on } \Gamma_{\sigma}^{N} .
\end{aligned}
$$

Thus, $W^{N}$ is the solution to the problem

$$
\begin{aligned}
L_{\varepsilon}^{N} W^{N}\left(x_{i}, t_{j}\right) & =0 & & \text { in } D_{\sigma}^{N}, \\
W^{N}\left(x_{i}, t_{j}\right) & =w\left(x_{i}, t_{j}\right) & & \text { on } \Gamma_{\sigma}^{N} .
\end{aligned}
$$

The error in our numerical solution can now also be decomposed

$$
\left(U^{N}-u\right)\left(x_{i}, t_{j}\right)=\left(\left(V^{N}-v\right)+\left(W^{N}-w\right)\right)\left(x_{i}, t_{j}\right) \quad \forall\left(x_{i}, t_{j}\right) \in \overline{D_{\sigma}^{N}},
$$

and we estimate the error in the regular component and the singular component separately.

Theorem 5.1 (Error in the Regular Component). Assume (2.2). The error in the regular component satisfies the following $\varepsilon$-uniform error bound

$$
\left|\left(V^{N}-v\right)\left(x_{i}, t_{j}\right)\right| \leqslant C\left(N_{x}^{-1}+N_{t}^{-1}\right) \quad \forall\left(x_{i}, t_{j}\right) \in \overline{D_{\sigma}^{N}},
$$

where $V^{N}$ is the solution of (5.1) and $v$ is the solution of (3.1). 
Proof. This can be established using the standard stability and consistency argument and the bounds given in (2.2).

Note that the above theorem is also valid on a uniform mesh. It is only when we come to deal with the singular component that the fitted mesh is needed. In order to examine the error in the singular component we will require the following bound:

$$
\left(1+\frac{2 \ln M}{M}\right)^{-M / 2} \leqslant \frac{2}{M} \quad \forall M \geqslant 4 .
$$

Theorem 5.2 (Error in the Singular Component). Assume (2.1). For all $N_{x} \geqslant 4$ and $N_{t}>\max _{\bar{D}}\{0,(2-d) / b\}$ we have the estimate

$$
\left|\left(W^{N}-w\right)\left(x_{i}, t_{j}\right)\right| \leqslant C\left(N_{x}^{-1}\left(\ln N_{x}\right)^{2}+N_{t}^{-1}\right) \quad \forall\left(x_{i}, t_{j}\right) \in \overline{D_{\sigma}^{N}},
$$

where $W^{N}$ is the solution of (5.2) and $w$ is the solution of (3.2).

Proof. We consider separately the cases $\sigma=1 / 2$ and $\sigma=\sqrt{\varepsilon} \ln N_{x}$. In the first case the mesh is uniform and we have

$$
\varepsilon^{-1} \leqslant 4\left(\ln N_{x}\right)^{2}
$$

We can derive an equivalent expression for the truncation error as for the regular component. Using the bounds on the derivatives of $w$ (which follow from the bounds on the derivatives of $u$ given in (2.1)), (5.4), and the fact that $x_{i+1}-x_{i-1}=2 N_{x}^{-1}, x_{i+1}-x_{i}=N_{x}^{-1}$ and $t_{j}-t_{j-1}=N_{t}^{-1}$, we get

$$
\begin{aligned}
\left|L_{\varepsilon}^{N}\left(W^{N}-w\right)\left(x_{i}, t_{j}\right)\right| & \leqslant C\left(N_{x}^{-1} \varepsilon^{-1 / 2}+N_{x}^{-1} \varepsilon^{-1}+N_{t}^{-1}\right) \\
& \leqslant C\left(N_{x}^{-1}\left(\ln N_{x}\right)^{2}+N_{t}^{-1}\right) \quad \forall\left(x_{i}, t_{j}\right) \in D_{\sigma}^{N} .
\end{aligned}
$$

Applying Lemma 4.2 then gives

$$
\left|\left(W^{N}-w\right)\left(x_{i}, t_{j}\right)\right| \leqslant C\left(N_{x}^{-1}\left(\ln N_{x}\right)^{2}+N_{t}^{-1}\right) \quad \forall\left(x_{i}, t_{j}\right) \in \overline{D_{\sigma}^{N}}
$$

We now consider the other case, that is, when $\sigma=\sqrt{\varepsilon} \ln N_{x}$. The mesh is now piecewise uniform and the mesh spacing (in the $x$ direction) is $h=2 \sigma / N_{x}$ in the subinterval $(0, \sigma)$ and $H=2(1-\sigma) / N_{x}$ in the subinterval $(\sigma, 1)$. The argument now depends on the position of the mesh point $x_{i}$.

If $x_{i} \in[\sigma, 1)$, then from the bound on $w$ given in Theorem 3.1 we have

$$
\left|w\left(x_{i}, t_{j}\right)\right| \leqslant C e^{-\sigma / \sqrt{\varepsilon}}=C N_{x}^{-1}
$$

To derive a similar bound on $W^{N}$ we introduce the mesh function $Y^{N}$ which is defined as follows

$$
Y_{i, j}^{N} \equiv Y^{N}\left(x_{i}, t_{j}\right)= \begin{cases}\left(1+\frac{h}{\sqrt{\varepsilon}}\right)^{-i}\left(1-\frac{A}{N_{t}}\right)^{-j}, & 0 \leqslant i \leqslant \frac{N_{x}}{2} \\ Y_{N_{x} / 2, j}^{N}\left(1+\frac{H}{\sqrt{\varepsilon}}\right)^{-\left(i-N_{x} / 2\right)}\left(1-\frac{A}{N_{t}}\right)^{-j}, & \frac{N_{x}}{2}<i \leqslant N_{x}\end{cases}
$$

where $A=\max _{\bar{D}}\{0,(2-d) / b\}$. 
Provided we choose $N_{t}$ large enough (i.e., $N_{t}>A$ ), we have

$$
Y^{N}\left(x_{i}, t_{j}\right) \geqslant 0 \quad \forall\left(x_{i}, t_{j}\right) \in \overline{D_{\sigma}^{N}} .
$$

Also,

$$
D_{x}^{+} Y\left(x_{i}, t_{j}\right)<0, \quad D_{t}^{-} Y\left(x_{i}, t_{j}\right)>0 \quad \forall\left(x_{i}, t_{j}\right) \in D_{\sigma}^{N} .
$$

Now we apply the difference operator to $Y^{N}$ :

$$
\begin{aligned}
L_{\varepsilon}^{N} Y_{i, j}^{N} & =\varepsilon \delta_{x}^{2} Y_{i, j}^{N}+a\left(x_{i}, t_{j}\right) D_{x}^{+} Y_{i, j}^{N}-b\left(x_{i}, t_{j}\right) D_{t}^{-} Y_{i, j}^{N}-d\left(x_{i}, t_{j}\right) Y_{i, j}^{N} \\
& \leqslant \varepsilon \delta_{x}^{2} Y_{i, j}^{N}+a\left(x_{i}, t_{j}\right) D_{x}^{+} Y_{i, j}^{N} .
\end{aligned}
$$

Depending on the position of the mesh point $x_{i}$ we get two different expressions. If $i \neq N_{x} / 2$ we have

$$
L_{\varepsilon}^{N} Y_{i, j} \leqslant(1-A b-d) Y_{i, j}^{N} \leqslant 0 \quad \forall j
$$

While if $i=N_{x} / 2$

$$
L_{\varepsilon}^{N} Y_{N_{x} / 2, j} \leqslant\left(N_{x} H-A b-d\right) Y_{i, j}^{N} \leqslant(2-A b-d) Y_{i, j}^{N} \leqslant 0 \quad \forall j .
$$

Now consider the mesh function $C Y^{N}-W^{N}$. We have

$$
\left(C Y^{N}-W^{N}\right)\left(x_{i}, t_{j}\right) \geqslant 0 \quad \forall\left(x_{i}, t_{j}\right) \in \Gamma_{\sigma}^{N},
$$

once we take

$$
C \geqslant \max _{0 \leqslant j \leqslant N_{t}}\left|W^{N}\left(0, t_{j}\right)\right|
$$

Also,

$$
L_{\varepsilon}^{N}\left(C Y^{N}-W^{N}\right)\left(x_{i}, t_{j}\right)=\left(C L_{\varepsilon}^{N} Y^{N}-L_{\varepsilon}^{N} W^{N}\right)\left(x_{i}, t_{j}\right)=C L_{\varepsilon}^{N} Y^{N}\left(x_{i}, t_{j}\right) \leqslant 0 .
$$

Then Lemma 4.1 applies and we get

$$
W^{N}\left(x_{i}, t_{j}\right) \leqslant C Y^{N}\left(x_{i}, t_{j}\right) \quad \forall\left(x_{i}, t_{j}\right) \in \overline{D_{\sigma}^{N}} .
$$

But

$$
Y^{N}\left(N_{x} / 2, t_{j}\right)=\left(1+\frac{2 \ln N_{x}}{N_{x}}\right)^{-N_{x} / 2}\left(1-\frac{A}{N_{t}}\right)^{-j} \leqslant 2 N_{x}^{-1}\left(1-\frac{A}{N_{t}}\right)^{-N_{t}} \leqslant C N_{x}^{-1},
$$

by (5.3) and the fact that

$$
\lim _{N_{t} \rightarrow \infty}\left(1-\frac{A}{N_{t}}\right)^{-N_{t}} \leqslant C
$$

Therefore we get

$$
W^{N}\left(x_{i}, t_{j}\right) \leqslant C N_{x}^{-1} \quad \forall\left(x_{i}, t_{j}\right) \in \overline{D_{\sigma}^{N}} \quad \text { s.t. } \quad x_{i} \in[\sigma, 1),
$$

and the error in the singular component in this case can now be estimated as

$$
\begin{aligned}
\left|\left(W^{N}-w\right)\left(x_{i}, t_{j}\right)\right| & \leqslant\left|W^{N}\left(x_{i}, t_{j}\right)\right|+\left|w\left(x_{i}, t_{j}\right)\right| \\
& \leqslant C N_{x}^{-1} \quad \forall\left(x_{i}, t_{j}\right) \in \overline{D_{\sigma}^{N}} \quad \text { s.t. } \quad x_{i} \in[\sigma, 1) .
\end{aligned}
$$


If $x_{i} \in(0, \sigma)$, then the expression for our truncation error becomes

$$
\left|L_{\varepsilon}^{N}\left(W^{N}-w\right)\left(x_{i}, t_{j}\right)\right| \leqslant C\left(N_{x}^{-1} \ln N_{x}+a\left(x_{i}, t_{j}\right) N_{x}^{-1} \ln N_{x} \varepsilon^{-1 / 2}+N_{t}^{-1}\right) .
$$

Now consider the discrete barrier functions

$$
\psi^{ \pm}\left(x_{i}, t_{j}\right)=C\left(N_{x}^{-1} \ln N_{x} \varepsilon^{-1 / 2}\left(\sigma-x_{i}\right)+\left(N_{x}^{-1}\left(\ln N_{x}\right)+N_{t}^{-1}\right) t_{j}+N_{x}^{-1}\right) \pm\left(W^{N}-w\right)\left(x_{i}, t_{j}\right) .
$$

Applying Lemma 4.2 in the subregion of $\overline{D_{\sigma}^{N}}$ bounded by $t=0, x=0$, and $x=\sigma$ we get

$$
\begin{aligned}
\psi_{i, j}^{ \pm} & \geqslant 0 \quad \forall\left(x_{i}, t_{j}\right) \in \overline{D_{\sigma}^{N}} \quad \text { s.t. } \quad x_{i} \in(0, \sigma), \\
\Rightarrow\left|\left(W^{N}-w\right)\left(x_{i}, t_{j}\right)\right| & \leqslant C\left(N_{x}^{-1}\left(\ln N_{x}\right) \varepsilon^{-1 / 2} \sigma+\left(N_{x}^{-1}\left(\ln N_{x}\right)+N_{t}^{-1}\right) t_{j}+N_{x}^{-1}\right) \\
& \leqslant C\left(N_{x}^{-1}\left(\ln N_{x}\right)^{2}+N_{t}^{-1}\right) \quad \forall\left(x_{i}, t_{j}\right) \in \overline{D_{\sigma}^{N}} \quad \text { s.t. } x_{i} \in(0, \sigma) .
\end{aligned}
$$

Combining the estimates in each subregion gives us the required result

$$
\left|\left(W^{N}-w\right)\left(x_{i}, t_{j}\right)\right| \leqslant C\left(N_{x}^{-1}\left(\ln N_{x}\right)^{2}+N_{t}^{-1}\right) \quad \forall\left(x_{i}, t_{j}\right) \in \overline{D_{\sigma}^{N}}
$$

The previous two theorems together give us the following $\varepsilon$-uniform estimate of the error in our numerical approximation at the mesh points.

Theorem 5.3. Assume (2.1) and (2.2). For all $N_{x} \geqslant 4$ and $N_{t}>\max _{\bar{D}}\{0,(2-d) / b\}$

$$
\sup _{0<\varepsilon \leqslant 1}\left\|U^{N}-u\right\|_{\overline{D_{\sigma}^{N}}} \leqslant C\left(N_{x}^{-1}\left(\ln N_{x}\right)^{2}+N_{t}^{-1}\right)
$$

where $C$ is a constant independent of $N_{x}, N_{t}$, and $\varepsilon$.

\section{Numerical results}

In this section we present numerical results that verify computationally the theoretical result of the last section. We take $T=1$ and consider the following problem (for various values of $p \geqslant 1$ ) from problem class $P_{\varepsilon, p}$

\section{Problem 1.}

$$
\begin{aligned}
& \left(\varepsilon u_{x x}+x^{p} u_{x}-u_{t}-u\right)(x, t)=x^{2}-1 \quad \text { in } \quad D \text {, } \\
& u(0, t)=1+t^{2}, \quad u(1, t)=0, \quad 0 \leqslant t \leqslant 1, \\
& u(x, 0)=(1-x)^{2}, \quad 0 \leqslant x \leqslant 1 .
\end{aligned}
$$

We let $N_{x}=N_{t}=N$ and tabulate the computed errors, $E_{\varepsilon}^{N}$, and the computed $\varepsilon$ uniform errors, $E^{N}$, for a variety of values of $\varepsilon$ and $N$ for $p=1.0$ (Table 1 ). Note that the vertical dot notation indicates that in each column the errors have stabilized and remain essentially constant for each of the values of $\varepsilon$ omitted. From this table one can see how the error changes as $N$ increases for not too large values of $N$, and also the behavior of the error as the parameter $\varepsilon$ varies. For other values of $p$ the error behavior is analogous (Table 2). As we do not have an exact solution for the above problem, we use the piecewise 
bilinear interpolant of the numerical solution on the finest available mesh, viz. $\bar{U}^{1024}$ as an approximation to the exact solution. Thus, we define $E_{\varepsilon}^{N}$ and $E_{\varepsilon}$ as follows

$$
E_{\varepsilon}^{N}=\max _{0 \leqslant i, j \leqslant N}\left|U^{N}\left(x_{i}, t_{j}\right)-\bar{U}^{1024}\left(x_{i}, t_{j}\right)\right|, \quad E^{N}=\max _{\varepsilon=1,2^{-1}, \ldots, 2^{-32}} E_{\varepsilon}^{N} .
$$

We also tabulate the computed $\varepsilon$-uniform orders of convergence $q^{N}$ for each value of $p$ considered (Table 3). These are calculated from the two-mesh differences defined as

$$
D_{\varepsilon}^{N}=\max _{0 \leqslant i, j \leqslant N}\left|U^{N}\left(x_{i}, t_{j}\right)-\bar{U}^{2 N}\left(x_{i}, t_{j}\right)\right|, \quad D^{N}=\max _{\varepsilon=1,2^{-1}, \ldots, 2^{-32}} D_{\varepsilon}^{N} .
$$

The $q^{N}$ are then defined as

$$
q^{N}=\log _{2} \frac{D^{N}}{D^{2 N}}
$$

\begin{tabular}{|c|c|c|c|c|c|c|}
\hline \multirow[b]{2}{*}{$\varepsilon$} & \multicolumn{6}{|c|}{ Number of Intervals $N$} \\
\hline & 8 & 16 & 32 & 64 & 128 & 256 \\
\hline 1.0 & $3.44 \mathrm{e}-02$ & $2.01 \mathrm{e}-02$ & $1.15 \mathrm{e}-02$ & $5.95 \mathrm{e}-03$ & $2.88 \mathrm{e}-03$ & $1.26 \mathrm{e}-03$ \\
\hline $2^{-1}$ & $85 \mathrm{e}-02$ & $9.46 \mathrm{e}-03$ & $4.99 \mathrm{e}-03$ & $2.54 \mathrm{e}-03$ & $1.22 \mathrm{e}-03$ & $5.28 \mathrm{e}-04$ \\
\hline $2^{-2}$ & $2.89 \mathrm{e}-02$ & $1.46 \mathrm{e}-02$ & $7.23 \mathrm{e}-03$ & $3.52 \mathrm{e}-03$ & $1.64 \mathrm{e}-03$ & $7.06 \mathrm{e}-04$ \\
\hline $2^{-3}$ & $3.91 \mathrm{e}-02$ & $1.99 \mathrm{e}-02$ & $9.86 \mathrm{e}-03$ & $4.80 \mathrm{e}-03$ & $2.25 \mathrm{e}-03$ & $9.64 \mathrm{e}-04$ \\
\hline $2^{-4}$ & $4.97 \mathrm{e}-02$ & $2.51 \mathrm{e}-02$ & $1.25 \mathrm{e}-02$ & $6.07 \mathrm{e}-03$ & $2.84 \mathrm{e}-03$ & $1.22 \mathrm{e}-03$ \\
\hline $2^{-5}$ & $5.09 \mathrm{e}-02$ & $3.04 \mathrm{e}-02$ & $1.53 \mathrm{e}-02$ & $7.44 \mathrm{e}-03$ & $3.48 \mathrm{e}-03$ & $1.49 \mathrm{e}-03$ \\
\hline $2^{-6}$ & $4.93 \mathrm{e}-02$ & $2.93 \mathrm{e}-02$ & $1.68 \mathrm{e}-02$ & $9.18 \mathrm{e}-03$ & $4.29 \mathrm{e}-03$ & $1.84 \mathrm{e}-03$ \\
\hline $2^{-7}$ & $4.94 \mathrm{e}-02$ & $2.81 \mathrm{e}-02$ & $1.58 \mathrm{e}-02$ & 8.81e-03 & $4.67 \mathrm{e}-03$ & $2.28 \mathrm{e}-03$ \\
\hline $2^{-8}$ & $5.41 \mathrm{e}-02$ & $3.01 \mathrm{e}-02$ & $1.53 \mathrm{e}-02$ & $8.38 \mathrm{e}-03$ & $4.39 \mathrm{e}-03$ & $2.04 \mathrm{e}-03$ \\
\hline $2^{-9}$ & $5.70 \mathrm{e}-02$ & $3.19 \mathrm{e}-02$ & $1.66 \mathrm{e}-02$ & $8.22 \mathrm{e}-03$ & $4.31 \mathrm{e}-03$ & $2.01 \mathrm{e}-03$ \\
\hline $2^{-10}$ & $5.90 \mathrm{e}-02$ & $3.29 \mathrm{e}-02$ & $1.74 \mathrm{e}-02$ & $8.70 \mathrm{e}-03$ & $4.27 \mathrm{e}-03$ & $2.00 \mathrm{e}-03$ \\
\hline $2^{-11}$ & $6.02 \mathrm{e}-02$ & $3.37 \mathrm{e}-02$ & $1.81 \mathrm{e}-02$ & $9.08 \mathrm{e}-03$ & $4.31 \mathrm{e}-03$ & $1.99 \mathrm{e}-03$ \\
\hline $2^{-12}$ & $6.11 \mathrm{e}-02$ & $3.45 \mathrm{e}-02$ & $1.85 \mathrm{e}-02$ & $9.34 \mathrm{e}-03$ & $4.45 \mathrm{e}-03$ & $1.98 \mathrm{e}-03$ \\
\hline $2^{-13}$ & $6.16 \mathrm{e}-02$ & $3.50 \mathrm{e}-02$ & $1.88 \mathrm{e}-02$ & $9.51 \mathrm{e}-03$ & $4.55 \mathrm{e}-03$ & $1.98 \mathrm{e}-03$ \\
\hline $2^{-14}$ & $6.20 \mathrm{e}-02$ & $3.53 \mathrm{e}-02$ & $1.90 \mathrm{e}-02$ & $9.65 \mathrm{e}-03$ & $4.62 \mathrm{e}-03$ & $2.01 \mathrm{e}-03$ \\
\hline $2^{-15}$ & $6.23 \mathrm{e}-02$ & $3.55 \mathrm{e}-02$ & $1.91 \mathrm{e}-02$ & $9.74 \mathrm{e}-03$ & $4.67 \mathrm{e}-03$ & $2.03 \mathrm{e}-03$ \\
\hline $2^{-16}$ & $6.24 \mathrm{e}-02$ & $3.57 \mathrm{e}-02$ & $1.92 \mathrm{e}-02$ & $9.80 \mathrm{e}-03$ & $4.71 \mathrm{e}-03$ & $2.05 \mathrm{e}-03$ \\
\hline $2^{-17}$ & $6.26 \mathrm{e}-02$ & $3.58 \mathrm{e}-02$ & $1.93 \mathrm{e}-02$ & $9.84 \mathrm{e}-03$ & $4.73 \mathrm{e}-03$ & $2.06 \mathrm{e}-03$ \\
\hline $2^{-18}$ & $6.26 \mathrm{e}-02$ & $3.59 \mathrm{e}-02$ & $1.93 \mathrm{e}-02$ & $9.87 \mathrm{e}-03$ & $4.75 \mathrm{e}-03$ & $2.07 \mathrm{e}-03$ \\
\hline $2^{-19}$ & $6.27 \mathrm{e}-02$ & $3.59 \mathrm{e}-02$ & $1.93 \mathrm{e}-02$ & $9.89 \mathrm{e}-03$ & $4.76 \mathrm{e}-03$ & $2.08 \mathrm{e}-03$ \\
\hline $2^{-20}$ & $6.28 \mathrm{e}-02$ & $3.60 \mathrm{e}-02$ & $1.94 \mathrm{e}-02$ & $9.90 \mathrm{e}-03$ & $4.77 \mathrm{e}-03$ & $2.08 \mathrm{e}-03$ \\
\hline $2^{-21}$ & $6.28 \mathrm{e}-02$ & $3.60 \mathrm{e}-02$ & $1.94 \mathrm{e}-02$ & $9.91 \mathrm{e}-03$ & $4.78 \mathrm{e}-03$ & $2.08 \mathrm{e}-03$ \\
\hline $2^{-22}$ & $6.28 \mathrm{e}-02$ & $3.60 \mathrm{e}-02$ & $1.94 \mathrm{e}-02$ & $9.92 \mathrm{e}-03$ & $4.78 \mathrm{e}-03$ & $2.09 \mathrm{e}-03$ \\
\hline $2^{-23}$ & $6.28 \mathrm{e}-02$ & $3.60 \mathrm{e}-02$ & $1.94 \mathrm{e}-02$ & $9.93 \mathrm{e}-03$ & $4.78 \mathrm{e}-03$ & $2.09 \mathrm{e}-03$ \\
\hline $2^{-24}$ & $6.28 \mathrm{e}-02$ & $3.61 \mathrm{e}-02$ & $1.94 \mathrm{e}-02$ & $9.93 \mathrm{e}-03$ & $4.79 \mathrm{e}-03$ & $2.09 \mathrm{e}-03$ \\
\hline : & : & $:$ & : & $\cdot$ & & \\
\hline $2^{-32}$ & $6.29 \mathrm{e}-02$ & $3.61 \mathrm{e}-02$ & $1.94 \mathrm{e}-02$ & $9.94 \mathrm{e}-03$ & $4.79 \mathrm{e}-03$ & $2.09 \mathrm{e}-03$ \\
\hline$E^{N}$ & $6.29 \mathrm{e}-02$ & $3.61 \mathrm{e}-02$ & $1.94 \mathrm{e}-02$ & $9.94 \mathrm{e}-03$ & $4.79 \mathrm{e}-03$ & $2.28 \mathrm{e}-03$ \\
\hline
\end{tabular}

Table 1. Table of computed errors $E_{\varepsilon}^{N}$ and $E^{N}$ for Problem 1 with $p=1.0$ 
We see from Table 1 that the values of $E_{\varepsilon}^{N}$ for each fixed $\varepsilon$ decrease with increasing $N$. $A$ fortiori the values of $E^{N}$ decrease with increasing $\mathrm{N}$ indicating that the convergence is uniform with respect to $\varepsilon$. For various values of $p, \varepsilon$-uniform orders of convergence depending on $N$ are given in Table 3. As $N$ increases, the convergence order approaches 1, which corresponds to the conclusion of Theorem 5.3. For this particular problem, the orders of convergence (over the range of $N \in[8,256]$ ) are higher than the theoretical rate given in Theorem 5.3 (see [2] for some sample values). The orders tend to the order associated with $C N^{-1} \ln N$ for all values of $p$. In Fig. 1 we plot the numerical solution of Problem 1 for a particular value of $p, \varepsilon$ and $N$.

Table 2. Table of computed $\varepsilon$-uniform errors $E^{N}$ for Problem 1 for various values of $p$

\begin{tabular}{||c||c|c|c|c|c|c||}
\hline \multicolumn{1}{||c||}{} & \multicolumn{7}{c||}{ Number of Intervals $N$} \\
\cline { 2 - 7 }$p$ & 8 & 16 & 32 & 64 & 128 & 256 \\
\hline 2.0 & $5.67 \mathrm{e}-02$ & $4.09 \mathrm{e}-02$ & $2.28 \mathrm{e}-02$ & $1.21 \mathrm{e}-02$ & $5.96 \mathrm{e}-03$ & $2.64 \mathrm{e}-03$ \\
\hline 3.0 & $6.40 \mathrm{e}-02$ & $4.12 \mathrm{e}-02$ & $2.43 \mathrm{e}-02$ & $1.33 \mathrm{e}-02$ & $6.68 \mathrm{e}-03$ & $2.99 \mathrm{e}-03$ \\
\hline 5.0 & $7.09 \mathrm{e}-02$ & $4.60 \mathrm{e}-02$ & $2.68 \mathrm{e}-02$ & $1.49 \mathrm{e}-02$ & $7.58 \mathrm{e}-03$ & $3.43 \mathrm{e}-03$ \\
\hline 10.0 & $4.39 \mathrm{e}-02$ & $3.72 \mathrm{e}-02$ & $2.83 \mathrm{e}-02$ & $1.65 \mathrm{e}-02$ & $8.63 \mathrm{e}-03$ & $4.01 \mathrm{e}-03$ \\
\hline
\end{tabular}

Table 3. Table of computed $\varepsilon$-uniform orders of convergence $q^{N}$ for Problem 1 for various values of $p$

\begin{tabular}{||c||c|c|c|c|c|c||}
\hline \multicolumn{1}{||c||}{} & \multicolumn{7}{c||}{ Number of Intervals $N$} \\
\cline { 2 - 7 } & 8 & 16 & 32 & 64 & 128 & 256 \\
\hline 1.0 & $6.77 \mathrm{e}-01$ & $8.11 \mathrm{e}-01$ & $8.85 \mathrm{e}-01$ & $9.35 \mathrm{e}-01$ & $8.89 \mathrm{e}-01$ & $8.97 \mathrm{e}-01$ \\
\hline 2.0 & $3.88 \mathrm{e}-01$ & $7.44 \mathrm{e}-01$ & $8.25 \mathrm{e}-01$ & $8.95 \mathrm{e}-01$ & $9.37 \mathrm{e}-01$ & $9.62 \mathrm{e}-01$ \\
\hline 3.0 & $5.84 \mathrm{e}-01$ & $5.80 \mathrm{e}-01$ & $7.87 \mathrm{e}-01$ & $8.72 \mathrm{e}-01$ & $9.19 \mathrm{e}-01$ & $9.51 \mathrm{e}-01$ \\
\hline 5.0 & $3.79 \mathrm{e}-01$ & $6.00 \mathrm{e}-01$ & $7.81 \mathrm{e}-01$ & $8.04 \mathrm{e}-01$ & $8.96 \mathrm{e}-01$ & $9.33 \mathrm{e}-01$ \\
\hline 10.0 & $1.15 \mathrm{e}-01$ & $5.11 \mathrm{e}-01$ & $5.94 \mathrm{e}-01$ & $7.34 \mathrm{e}-01$ & $8.52 \mathrm{e}-01$ & $9.07 \mathrm{e}-01$ \\
\hline
\end{tabular}

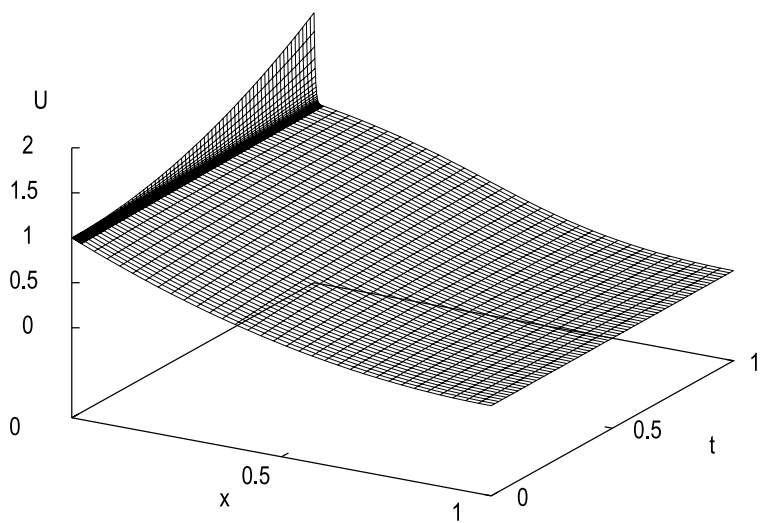

Figure 1. Numerical solutions for Problem 1 with $p=2, N=64$ and $\varepsilon=2^{-15}$ 


\section{References}

[1] R. K. Dunne, E. O'Riordan, and G. I. Shishkin, Singularly perturbed parabolic problems on nonrectangular domains, Numerical Analysis and Its Applications, Second International Conference, NAA 2000, Rousse, Bulgaria, June 2000 (L. Vulkov, J. Waśniewski, and P. Yalamov, eds.), Springer-Verlag, 2001, Lecture Notes in Computer Science, 1988, pp. 265-272.

[2] P. A. Farrell, A. F. Hegarty, J. J. H. Miller, E. O'Riordan, and G. I. Shishkin, Robust Computational Techniques for Boundary Layers, Chapman and Hall/CRC Press, Boca Raton, USA, 2000.

[3] N. V. Kopteva, Uniform pointwise convergence of difference schemes for convection-diffusion problems on layer-adapted meshes, Computing, 66 (2001), No. 2, pp. 179-197.

[4] O. A. Ladyzhenskaya, V. A. Solonnikov, and N. N. Ural'tseva, Linear and Quasilinear Equations of Parabolic Type, Translations of Mathematical Monographs, Vol. 23, American Mathematical Society, USA, 1968.

[5] T. Linß, Solution decomposition for convection-diffusion problems with boundary turning points and an application, Preprint MATH-NM-05-01, 2001.

[6] V. D. Liseikin, On the numerical solution of singularly perturbed problems with turning points, Comput. Math. Math. Phys., 41 (2001), No. 1, pp. 55-83.

[7] J. J. H. Miller, E. O'Riordan, and G. I. Shishkin, Fitted numerical methods for singular perturbation problems, World Scientific Publishing Co., Singapore, 1996.

[8] J. J. H. Miller, E. O'Riordan, G. I. Shishkin, and L. P. Shishkina, Fitted mesh methods for problems with parabolic boundary layers, Mathematical Proceedings of the Royal Irish Academy, 98A (1998), No. 2, pp. 173-190.

[9] H. -G. Roos, M. Stynes, and L. Tobiska, Numerical Methods for Singularly Perturbed Differential Equations. Convection-Diffusion and Flow Problems, Springer-Verlag, New York, 1996.

[10] G. I. Shishkin, Discrete Approximation of Singularly Perturbed Elliptic and Parabolic Equations, Russian Academy of Sciences, Ural Section, Ekaterinburg, 1992.

[11] M. Stynes and E. O'Riordan, Uniformly convergent difference schemes for singularly perturbed parabolic diffusion-convection problems without turning points, Numer. Math., 55 (1989), pp. 521-544.

[12] R. Vulanović and P. A. Farrell, Analysis of multiple turning point problems, Rad. Mat., 8 (1992).

[13] R. Vulanović and P. A. Farrell, Continuous and numerical analysis of a multiple turning point problem, SIAM J. Numer. Anal., 30 (1993), No. 5, pp. 1400-1418. 